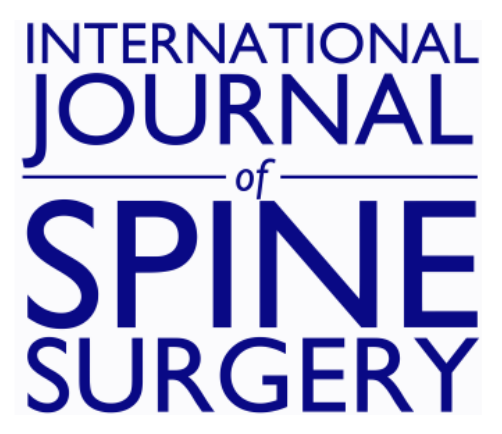

\title{
Load Share Mapping for Traditional PEEK vs Novel Hybrid PEEK With Expandable Porous Mesh Intervertebral Devices
}

Lisa A. Ferrara, Pierce D. Nunley and Marcus B. Stone

Int J Spine Surg 2020, 14 (s3) S115-S120

doi: https://doi.org/10.14444/7134

http://ijssurgery.com/content/14/s3/S115

This information is current as of April 26, 2023.

Email Alerts Receive free email-alerts when new articles cite this article. Sign up at:

http://ijssurgery.com/alerts

The International Journal of Spine Surgery

2397 Waterbury Circle, Suite 1,

Aurora, IL 60504, Phone: +1-630-375-1432 


\title{
Load Share Mapping for Traditional PEEK vs Novel Hybrid PEEK With Expandable Porous Mesh Intervertebral Devices
}

\author{
LISA A. FERRARA, PHD, ${ }^{1}$ PIERCE D. NUNLEY, MD ${ }^{2}$ MARCUS B. STONE, PHD ${ }^{2}$ \\ ${ }^{1}$ OrthoKinetic Technologies, Southport, North Carolina, ${ }^{2}$ Spine Institute of Louisiana, Shreveport, Louisiana
}

\begin{abstract}
Background: A successful intervertebral fusion requires biomechanical stability created by the structural support of the interbody device and loading of the bone graft material to accelerate mechanotransduction and bone remodeling. The objective of this study was to generate a quantitative map of the contact area and stress profile for 2 implant designs; a rigid monolithic polyetheretherketone (PEEK) lateral cage (MPLC), and a unique hybrid interbody design, which includes PEEK terminal supports surrounding an expandable porous mesh $(\mathrm{P}+\mathrm{EPM})$ that serves to contain bone graft.

Methods: The construct for each test consisted of a device sandwiched between 2 flat or shaped Grade 15 foam blocks. Pressure sensitive film and thin film sensors were placed between the device and each of the foam blocks. A series of each implant type was compressed at a rate $0.1 \mathrm{~mm} /$ second for 2 loads $(1100 \mathrm{~N}$ and $2000 \mathrm{~N})$ with and without bone graft. Device and bone graft contact area were analyzed for each test condition and corresponding load profiles were quantified and mapped.

Results: P+EPM demonstrated 34\% greater graft volume than MPLC resulting in a $28 \%$ larger area for bone exchange when filled. The load profiles for all applied loading paradigms for P+EPM demonstrated significant direct loading on the bone graft contained within the mesh, resulting in at least $170 \%$ greater loaded area than MPLC. Furthermore, the P+EPM demonstrated load sharing with the terminal PEEK supports. MPLC for all loading conditions demonstrated negligible bone graft loading.

Conclusions: $\mathrm{P}+\mathrm{EPM}$ allows for an optimized contact area for bone exchange and graft incorporation. The load profiles confirmed that the filled mesh does not stress shield terminal PEEK supports and will load share. The expandable, compliant, porous mesh provides a greater multiplanar area for bone exchange and allows for direct contact with the viscoelastic vertebral endplates, improving the endplate and graft interface mechanics.
\end{abstract}

Special Issue

Keywords: Dacron mesh, lumbar spine fusion, biomechanics, pressure testing

\section{INTRODUCTION}

Lumbar intervertebral fusion is a common spinal procedure to treat a range of debilitating spinal pathologies. Regardless of the surgical approach or fixation used for stabilization, the primary aim of this procedure is to correctly align and stabilize the spinal column in order to facilitate fusion. An intervertebral device is placed between the adjacent vertebral endplates along the anterior weightbearing axis of the spine to provide the immediate structural support to restore stability to the diseased segment and initiate bone healing (osseointegration) of the fusion graft construct. ${ }^{1-4}$ Additional anterior or posterior fixation is often used to further limit motion and increase rigidity across the fusion site.

In a reflection of Wolff's Law, compressive loads along the anterior column of the spine are transferred to the spinal motion segment housing the intervertebral fusion construct. Axial stresses incurred with motion are transferred along the spinal column and across the implanted fusion site, which can accelerate mechanotransduction in a biomechanically stable environment. ${ }^{4-6}$ The viscoelastic behavior of the vertebral endplates interfacing with the interbody construct can flex and respond in an elastic and viscous (fluid) manner where the basic mechanical principles of fluid flow help to transfer nutrients from the vertebral endplates into the graft material to promote bone healing. ${ }^{7,8}$ The interbody device housing the graft material provides the necessary structural support to resist these stresses. Consequently, ensuring that the bone graft contained within an intervertebral device is optimally loaded and not shielded from the necessary bone promoting stresses during the 

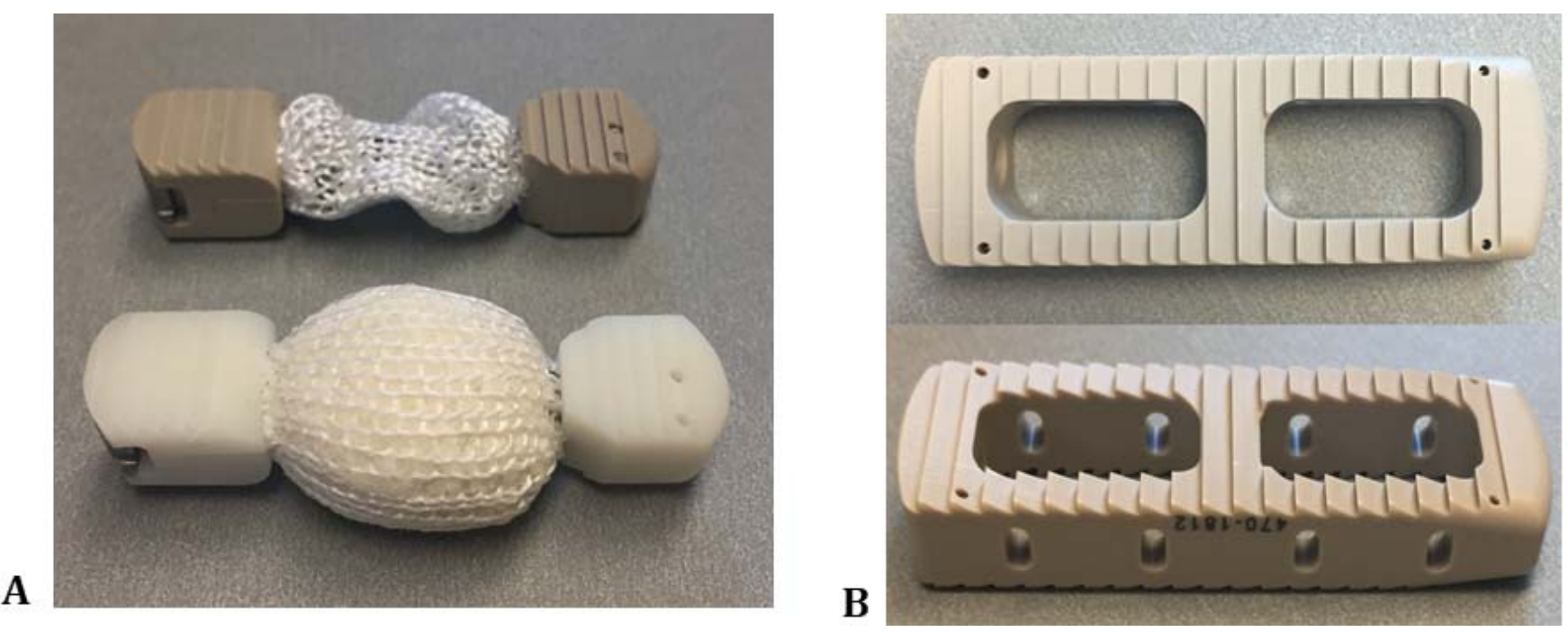

Figure 1. Representative images showing the P+EPM device (A) and the MPLC (B) device.

fusion process is a critical parameter in the design of these devices.

Many factors can contribute to the loss of the fixation integrity of a fusion site. ${ }^{1,9-11}$ Inadequate endplate preparation, patient selection, bone quality, and the surrounding biomechanical and physiological environments to name a few. However, optimization of interbody design features, functions, and footprint can minimize certain risks and require less work from the vertebral endplate viscoelastic properties to transport nutrients to the interspace and graft material in order to help promote fusion healing. ${ }^{3,4,7,12}$ A stable biomechanical environment early in the postoperative stage will allow for earlier osseointegration and less formation of fibrous tissue surrounding the graft site, thus improving the balance between stabilization and fusion healing. Combined, these factors have synergistic implications for intervertebral devices. The ideal device requires early stability to be accomplished, which can be achieved through implementing a large footprint with large bone communicating areas, conformity to the vertebral endplates to provide direct contact with the bone graft material, and structural strength to provide ample load bearing capacity. Open architectures

Table. Overview of the test plan.

\begin{tabular}{lclll}
\hline & \multicolumn{2}{c}{$\mathbf{P}+\mathbf{E P M}$} & & MPLC \\
\cline { 2 - 3 } Load [N] & Under-fill & Nominal Fill & & Nominal Fill \\
\hline Test block configuration & Flat & Flat, curved & & Flat, curved \\
1100 & 6 & 6 & 6 \\
2000 & 6 & 6 & 6 \\
\hline
\end{tabular}

with multiple planes of entry for bone ingrowth with large direct contact areas at the endplates for greater area for bone exchange are some of the design characteristics that may improve fusion healing.

The aim of this study was to map the bone graft contact area and respective stress profile for a novel expandable PEEK and porous mesh lateral interbody fusion device (P+EPM) and compare it with a traditional monolithic PEEK lateral interbody cage (MPLC). Two filling conditions (under-fill and nominal fill) were tested in order to evaluate the load sharing contribution of the grafts for the $\mathrm{P}+\mathrm{EPM}$, and only the fill condition for the MPLC was assessed.

\section{METHODS}

Six samples of the $12 \times 42 \mathrm{~mm}$ P+EPM (Figure 1A) and 6 of the $16 \times 42 \mathrm{~mm}$ MPLC (Figure 1B) were used for each test series for 2 compressive loading modes. The Table outlines the samples, sizes, loads, and fill conditions tested for each implant type. Additionally, 2 configurations of the test blocks were evaluated with the implants for the fill conditions only. Under fill configuration was only evaluated in flat test blocks.

Morselized bovine bone was used as fill for the graft materials and were filled per the manufacturer's specifications, with the exception of the underfill condition, which was filled to $66 \%$ of the recommended level. The construct for each test consisted of a device sandwiched between 2 grade 15 PU foam blocks (Pacific Research Laboratories, Inc, Vashon, WA) to simulate the vertebral end- 


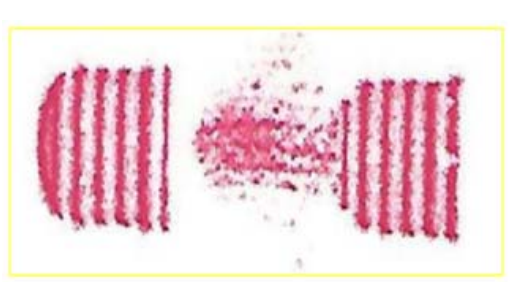

1

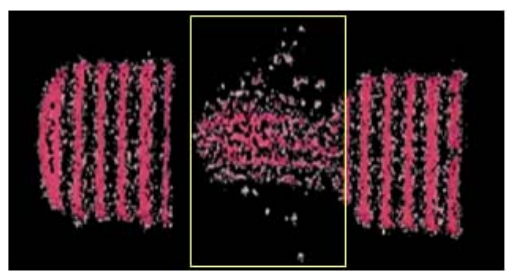

2

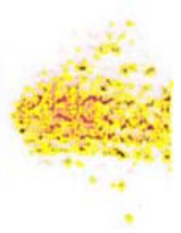

3

Figure 2. Procedure used to determine the contact footprint in ImageJ. First, the region of interest was selected (left), the image was then placed under threshold (middle), and the resultant area was selected and measured (right). The total footprint was determined by selecting the entire ROI (region of interest; left), then the graft footprint was measured by cropping the central area occupied by the graft (right). Implant footprint could then be determined as the difference between the total footprint and the graft footprint.

plates. Pressure sensitive film of low and medium threshold grade were initially analyzed to determine the appropriate pressure range for testing. The pressure film was placed between the superior and inferior endplates and foam bone blocks for each implant construct. The low-pressure film was identified as the appropriate range for all constructs tested (SensorTek pressure sensitive film, Sensor Products, Inc, Madison, NJ; FujiFilm Prescale and Topaq, SPI).

Testing was conducted on an MTS electromechanical materials test machine (MTS Corp, Eden Prairie, MN), and each construct was positioned such that the $Z$-axis of the device was aligned with the axis of the test machine's actuator and collinear with the load cell of the testing machine. Each of the filled interbody devices was compressed between the 2 foam bone blocks at a rate $0.1 \mathrm{~mm} /$ second for 3 consecutive applied compressive loads $(1100 \mathrm{~N}$, $2000 \mathrm{~N}, 3000 \mathrm{~N})$. The pressure sensitive film captured a visual impression and pressure profile of the loaded implant and bone graft regions and was changed at both the superior and inferior interfaces after each load application.

Area measurements were taken of the pressure film by thresholding the pressure film output in ImageJ (ImageJ Version 1.51k, National Institutes of Health, http://imagej.nih.gov/ij). Images were calibrated, cropped to the specified region of interest, and a threshold applied using a uniform value across all samples to isolate the contact footprint. The resultant represented the loaded portion of the graft footprint for each test condition and load level. Figure 2 illustrates this procedure. The total footprint was determined by selecting the entire region of interest (ROI) define (left), then the graft footprint was measured by cropping the central area occupied by the graft (right). Implant footprint could then be determined as the difference between the total footprint and the graft footprint through the thresholding procedure. Direct measurements by tracing the outline of the loaded graft portion were also taken to verify the threshold process (Figure 2).

\section{STATISTICAL METHODS}

Contact area for both device types were statistically compared using SPSS version 22 for windows. A 1-way analysis of variance with multiple posthoc comparisons was used to compare the areas of loaded bone graft detected by the pressure film data for the P+EPM and the MPLC fill configurations, both the flat and curved test blocks and the applied loads. A $P$ value of $<.001$ was considered statistically significant.

\section{RESULTS}

The P+EPM demonstrated a mean loaded bone graft area for $1100 \mathrm{~N}$ and $2000 \mathrm{~N}$ of $3.30 \pm 0.63 \mathrm{~cm}^{2}$ and $3.86 \pm 0.53 \mathrm{~cm}^{2}$, respectively for the flat blocks and $4.16 \pm 0.55 \mathrm{~cm}^{2}$ and $4.41 \pm 0.38 \mathrm{~cm}^{2}$ at $1100 \mathrm{~N}$ and $2000 \mathrm{~N}$, respectively for the curved block (Figure 3).

Pressure film impressions of the loaded bone graft areas were directly measured and then sent for pressure profile mapping (Figures 4 and 5). The mean areas for the loaded bone graft for $1100 \mathrm{~N}$ and $2000 \mathrm{~N}$ of $0.03 \pm 0.0 \mathrm{~cm}^{2}$ and $0.10 \pm 0.09 \mathrm{~cm}^{2}$, respectively for the flat blocks and $0.42+0.0 \mathrm{~cm}^{2}$ and $0.311 \pm 0.26 \mathrm{~cm}^{2}$ at $1100 \mathrm{~N}$ and $2000 \mathrm{~N}$, respectively for the curved blocks. The majority of the MPLC for both applied compressive loads did not register any loaded bone graft within the communicating areas of the interbody device. Therefore, the standard deviations for the MPLC reflect this, due to the low quantity of MPLC devices that did register areas less than $0.5 \mathrm{~cm}^{2}$ of loaded bone graft area.

The areas of the loaded bone graft communicating area for the MPLCs where negligible loading 


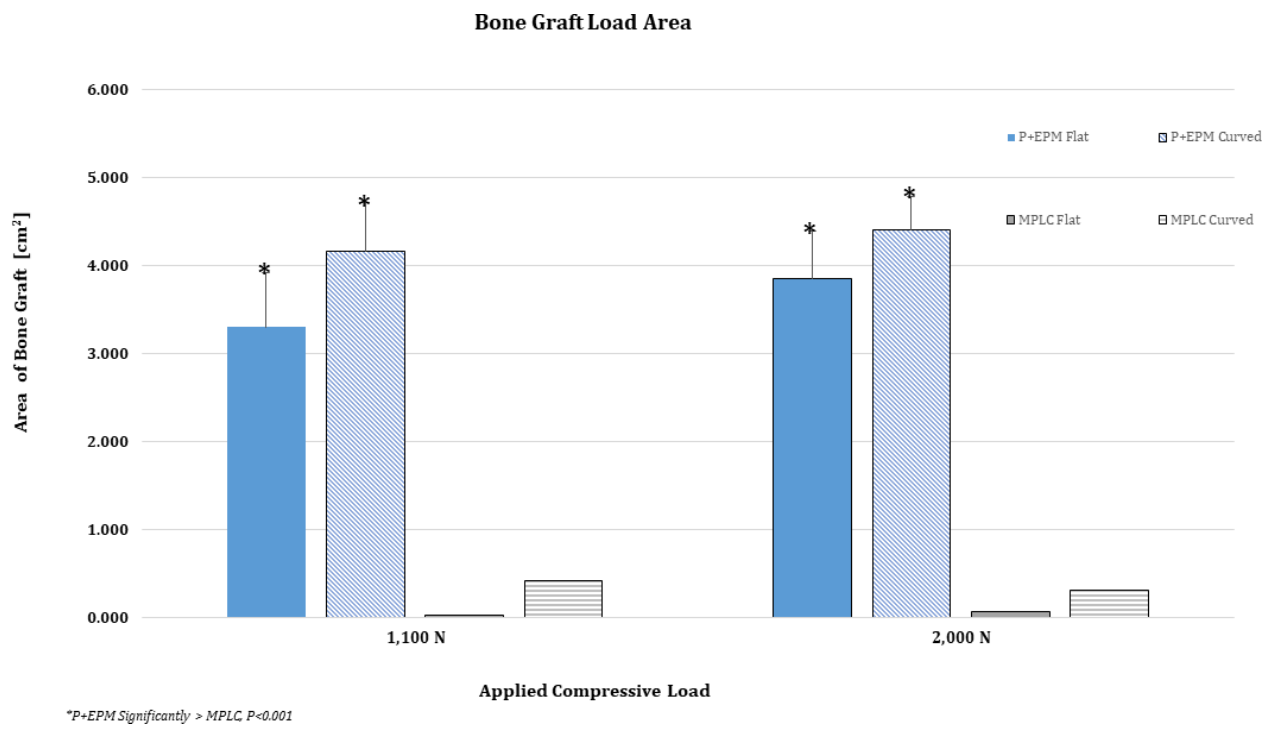

Figure 3. Measured bone graft areas under direct loading for $1100 \mathrm{~N}$ and $2000 \mathrm{~N}$ for the P+EPM versus MPLC. The measured areas of loaded bone graft for the $\mathrm{P}+$ EPM were significantly greater than the MPLC, $P<.001$. Very few samples tested at both loads for the MPLC did not detect direct loading of the bone graft material. There was 1 sample for the $1100 \mathrm{~N}$ and 2 samples for the $2000 \mathrm{~N}$ that detected bone graft loading less than $0.5 \mathrm{~cm}^{2}$.

A

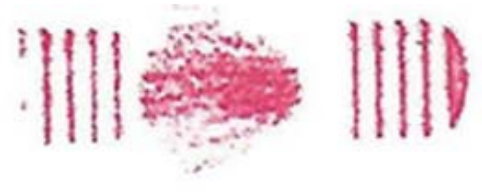

B
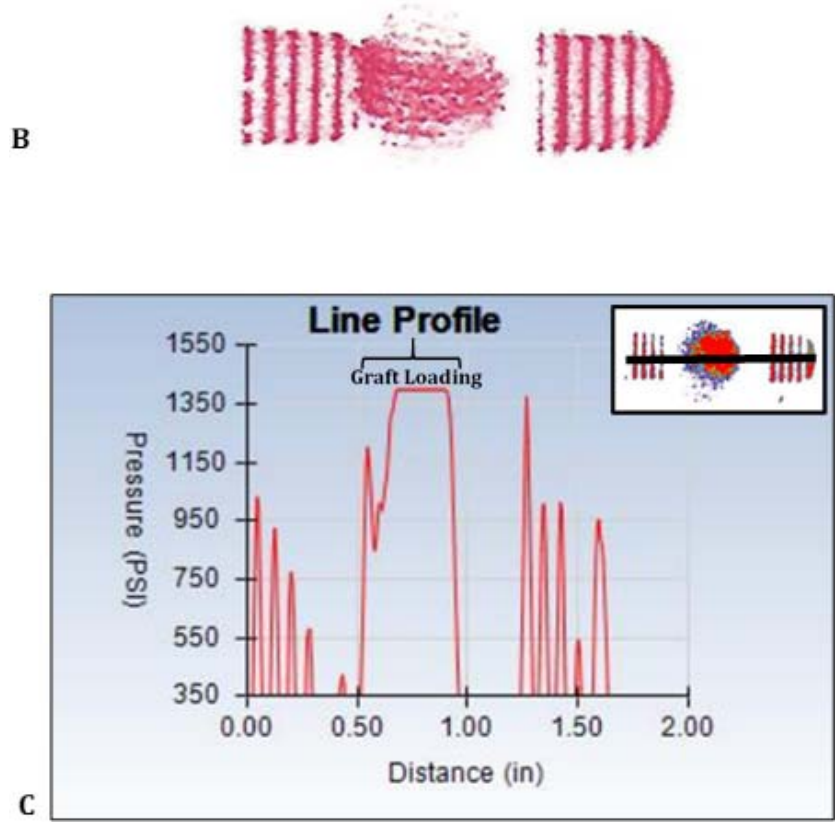

Figure 4. Central bone graft loading pressure maps measured for filled $\mathrm{P}+\mathrm{EPM}$ in flat Grade $15 \mathrm{pcf}$ blocks; under $1100 \mathrm{~N}$ load with pressures ranging from 3.0 MPa to 9.0 $\mathrm{MPa}(\mathrm{A})$, and under $2000 \mathrm{~N}$ load with pressures ranging from 3.4 MPa to $9.6 \mathrm{MPa}(\mathrm{B})$, and a representative pressure profile at $1100 \mathrm{~N}$ along the longitudinal axis of the P+EPM device. The pressure distribution through the P+EPM identifies that maximum pressures of $9.6 \mathrm{MPa}$ were measured within the bone graft region (bracket) with a mean pressure graft loading of $6.7 \mathrm{MPa}(\mathrm{C})$. was detected was significantly less than the areas measured for the P+EPM, $P<.001$ (Figure 4). There was no statistically significant difference between the test block types (flat or curved) or between the loads for the P+EPM.

The load profiles for the under fill and nominal fill conditions of the P+EPM demonstrated significantly greater graft contact area than the MPLC $(P$ $<.001)$. As the load was increased, the graft contact area increased for both the under fill and nominal fill configurations, indicative of the graft region (bone in the mesh) receiving axial loading. As the graft contact area increased, the load sharing between the bone graft region (filled mesh) and the PEEK terminal ends of the P+EPM further increased. This was reflected in the pressure impressions for both loads and block types. Additionally, these pressure profiles for both compressive loads demonstrated equivalent or greater measured pressures for the bone graft loaded regions than the terminal PEEK components of the device.

The MPLC pressure profiles demonstrated the lack of bone graft loading demonstrating a void in the measured pressures in the regions of the open bone communicating regions (Figure 5).

\section{DISCUSSION}

The under fill and nominal fill P+EPM demonstrated direct bone graft loading for all loads and configurations that exceeded that of conventional 


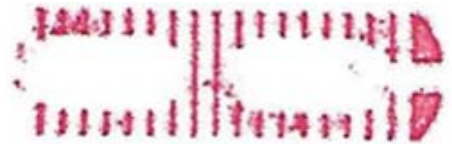
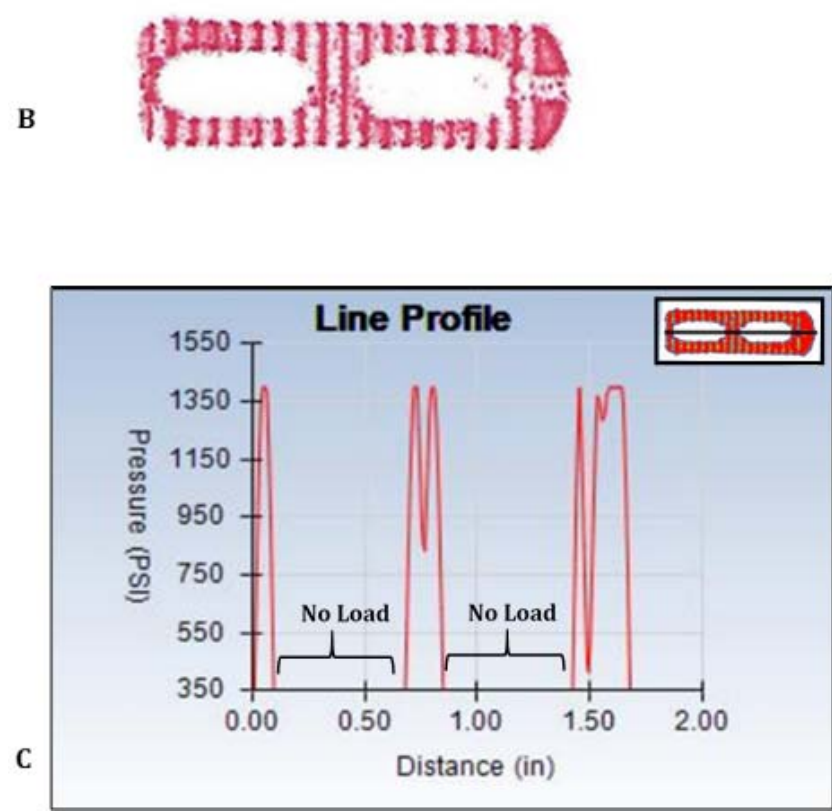

Figure 5. Evidence of central bone graft loading was not present in the central pores of the MPLC device in flat Grade 15 pcf blocks, as the flat blocks present the best case for potential loading of the bone graft region. The pressure maps at the central bone graft region of the MPLC registered as $0 \mathrm{MPa}$ for filled devices; under $1100 \mathrm{~N}$ load (A) and under $2000 \mathrm{~N}$ load, where $0 \mathrm{MPa}$ of pressure indicating the bone graft material in the central pores of the MPLC were not in direct contact of the vertebral endplates, nor directly loaded (B), and a representative pressure profile at $1100 \mathrm{~N}$ and also observed for $2000 \mathrm{~N}$, along the longitudinal axis of the P+EPM device (brackets indicate graft regions where loading of the graft material was not present). The pressure distribution through the MPLC measured within the bone graft cores did not measure any pressure $(0 \mathrm{MPa})$ indicating that the bone graft was not directly loaded under $1100 \mathrm{~N}$ and $2000 \mathrm{~N}(\mathrm{C})$.

monolithic interbody cages. The majority of the monolithic PEEK cages tested did not result in loading of the bone graft material. This would require the MPLC to rely upon the viscoelastic properties of the vertebral endplate to indirectly transfer loads to the bone graft and transport nutrients to the fusion site through fluid mechanics, where the endplate can flex about implant to indirectly load the interbody fusion graft to initiate mechanotransduction. ${ }^{4-6,13}$

The direct contact and large conforming surface area of the P+EPM with the vertebral endplates provide an advantage to achieving a balance between stabilization and time to heal. Direct immediate loading of the bone graft reduces the duration to obtain stabilization to allow for osseointegration. If the graft material is loaded shortly after implantation in a stable device, it will begin to load-share with the spinal segment. The open porous structure of the P+EPM in direct contact and conformity with the endplates provides a direct route for osseointegration with less work required by the vertebral endplates to transfer the stresses and nutrients to the graft site. ${ }^{6,14,15}$

Furthermore, the complete enclosure of the morselized bone graft material within the mesh of the $\mathrm{P}+\mathrm{EPM}$ allows for increased bone exchange from multiple planes. The morselized bone within the porous mesh provides a matrix for osseointegration along a significantly large surface area for bone exchange from multiple planes. The larger surface area of contact for the P+EPM distributes the force at the vertebral endplates over a larger area of the implant, resulting in lower stresses at the endplateimplant interface. ${ }^{3,4,7,12,16,17}$ It is well documented that an intervertebral device with a smaller footprint area would impose greater stress on the implant and would be of greater risk for subsidence.

Given the factors discussed above, the filled $\mathrm{P}+\mathrm{EPM}$ provides a large footprint area to reduce stresses at the vertebral endplate and the implant interface to reduce the risk to subsidence and provide early stability to the fusion site with immediate load sharing between the PEEK structural ends and graft areas of the implant. This results in a synergistic effect of promoting bone formation at the graft site, while distributing endplate loads over a greater area and minimizing potential stress shielding from the implant.

The MPLC was intentionally over packed and compacted with cancellous bone prior to the profile testing. After compression at $1100 \mathrm{~N}$ (lowest compressive load applied), the compacted cancellous bone in the MPLC was slightly less than the level of its open central pore. This phenomenon occurs with all MPLC interbody fusions (IBFs) as the bone is compressed into the device under high loads and/or the "topping off" effect for filling the cages is lost upon insertion into the disc space. At best, the filled monolithic cages start off where the bone is level with the edge of the central pores in these cages and would see less loading due to the surrounding PEEK material of these monolithic cages and is, therefore, shielding the graft material to a greater extent.

The study demonstrated that the PEEK structures are not stress shielding the bone graft component of the device and further showed that 
the loaded filled mesh shared the load with the PEEK terminal structures, analogous to how the structures of the spine share loads.

\section{CONCLUSIONS}

The compliant mesh of the P+EPM allows for greater contact area that stiffens with bone graft fill and conforms to the individual vertebral endplates, thus contributing to the overall structural support. Furthermore, the P+EPM provides greater multiplanar area for bone exchange with direct contact and conformability at the vertebral endplates to improve the micromechanics at the bone interface, while maintaining biomechanical stability across the fusion site. These characteristics should allow for more reliable fusion and patient outcomes. Clinical studies are currently ongoing and are anticipated to bear this out. Regardless of the specific implant selected, surgeons should consider how obtaining early biomechanical stability with interbody fixation can be employed to improve fusion success.

\section{REFERENCES}

1. Katsuura A, Hukuda S, Saruhashi Y, Mori K. Kyphotic malalignment after anterior cervical fusion is one of the factors promoting the degenerative process in adjacent intervertebral levels. Eur Spine J. 2001;10(4):320-324. doi:10.1007/s005860000243

2. Mummaneni PV, Haid RW, Rodts GE. Lumbar interbody fusion: state-of-the-art technical advances. $J$ Neurosurg Spine. 2004;1(1):24-30. doi:10.3171/spi.2004.1.1.0024

3. Weiner BK, Fraser RD. Spine update lumbar interbody cages. Spine (Phila Pa 1976). 1998;23(5):634-640. doi:10.1097/ 00007632-199803010-00020

4. Palepu V, Kiapour A, Goel VK, Moran JM. A unique modular implant system enhances load sharing in anterior cervical interbody fusion: a finite element study. Biomed Eng Online. 2014;13(1):26. doi:10.1186/1475-925X-13-26

5. Ledet EH, Sanders GP, DiRisio DJ, Glennon JC. Loadsharing through elastic micro-motion accelerates bone formation and interbody fusion. Spine J. 2018;18(7):1222-1230. doi:10.1016/j.spinee.2018.02.004

6. Willie BM, Yang X, Kelly NH, et al. Cancellous bone osseointegration is enhanced by loading. Tissue Eng Part $C$ Methods. 2010;16(6):1399-1406.

7. Ellingson AM, Nuckley DJ. Intervertebral disc viscoelastic parameters and residual mechanics spatially quantified using a hybrid confined/in situ indentation method. $J$ Biomech. 2012;45(3):491-496. doi:10.1016/j.jbiomech.2011.11.050

8. Liu J, Hao L, Suyou L, et al. Biomechanical properties of lumbar endplates and their correlation with MRI findings of lumbar degeneration. J Biomech. 2016;49(4):586-593. doi:10. 1016/j.jbiomech.2016.01.019
9. Ha SK, Park JY, Kim SH, Lim DJ, Kim SD, Lee SK. Radiologic assessment of subsidence in stand-alone cervical polyetheretherketone (PEEK) cage. J Korean Neurosurg Soc. 2008;44(6):370-374. doi:10.3340/jkns.2008.44.6.370

10. Epari DR, Kandziora F, Duda GN. Stress shielding in box and cylinder cervical interbody fusion cage designs. Spine (Phila Pa 1976). 2005;30(8):908-914. doi:10.1097/01.brs. 0000158971.74152.b6

11. Bartels RHMA, Donk RD, Feuth T. Subsidence of stand-alone cervical carbon fiber cages. Neurosurgery. 2006;58(3):502-507. doi:10.1227/01.NEU.0000197258.30821.50

12. Kandziora F, Schollmeier G, Scholz M, et al. Influence of cage design on interbody fusion in a sheep cervical spine model. J Neurosurg. 2002;96(Suppl 3):321-332. doi:10.3171/spi. 2002.96.3.0321

13. Middleton KAJ, Vivian Ma YH, You L. Measuring bone cell response to fluid shear stress and hydrostatic/dynamic pressure. In: Zdero R, ed. Experimental Methods in Orthopaedic Biomechanics. New York, NY: Elsevier, Inc; 2017:217-232. doi:10.1016/B978-0-12-803802-4.00014-7

14. Kayacan MC, Baykal YB, Karaaslan T, et al. Monitoring the osseointegration process in porous Ti6Al4V implants produced by additive manufacturing: an experimental study in sheep. J Appl Biomater Funct Mater. 2018;16(2):68-75. doi: $10.5301 / \mathrm{jabfm} .5000385$

15. Cooper LF, Masuda T, Yliheikkilä PK, Felton DA. Generalizations regarding the process and phenomenon of osseointegration. Part II. In vitro studies. Int J Oral Maxillofac Implants. 13(2):163-174. http://www.ncbi.nlm.nih.gov/pubmed/ 9581401. Accessed June 15, 2020.

16. MacLean JJ, Owen JP, Iatridis JC. Role of endplates in contributing to compression behaviors of motion segments and intervertebral discs. $J$ Biomech. 2007;40(1):55-63. doi:10.1016/j. jbiomech.2005.11.013

17. Rodriguez AG, Rodriguez-Soto AE, Burghardt AJ, Berven S, Majumdar S, Lotz JC. Morphology of the human vertebral endplate. J Orthop Res. 2012;30(2):280-287. doi:10. $1002 /$ jor. 21513

Disclosures and COI: The authors are paid consultants of Spineology, a company involved in the manufacture of a device examined in this study.

Corresponding Author: Marcus B. Stone, $\mathrm{PhD}$, Director of Clinical Research, Spine Institute of Louisiana, 1500 Line Ave, Suite 200, Shreveport, LA 71101-4643. Phone: 318-629-5585; Fax: 318629-6329; Email: mstone@louisianaspine.org.

Published 9 December 2020

This manuscript is generously published free of charge by ISASS, the International Society for the Advancement of Spine Surgery. Copyright (C) 2020 ISASS. To see more or order reprints or permissions, see http://ijssurgery.com. 